\title{
Efficacy and feasibility of day surgery using transurethral seminal vesiculoscopy under caudal block anesthesia for intractable hemospermia
}

\author{
Bo Cui, Jiang-Tao Wu, Jian-Jun Xu, Tong-Wen Ou^ \\ Department of Urology, Xuanwu Hospital, Capital Medical University, Beijing, China \\ Contributions: (I) Conception and design: B Cui, TW Ou; (II) Administrative support: TWO; (III) Provision of study materials or patients: B Cui, \\ TW Ou; (IV) Collection and assembly of data: B Cui, JT Wu, JJ Xu; (V) Data analysis and interpretation: B Cui, TW Ou; (VI) Manuscript writing: \\ All authors; (VII) Final approval of manuscript: All authors. \\ Correspondence to: Tong-Wen Ou. Department of Urology, Xuanwu Hospital, Capital Medical University, Beijing 100053, China. \\ Email: outongwen1967@126.com.
}

Background: Transurethral seminal vesiculoscopy (TSV) is a safe and effective method for intractable
hemospermia. It uses a natural cavity, is fast, involves little trauma, and boasts a low incidence of
complications. Because uncontrollable penile erection during general anesthesia will severely influence the
surgical operation or even damage the endoscope, spinal anesthesia was applied more on TSV. But spinal
anesthesia extends the length of stay in the hospital and brings patients unnecessary discomfort. As the TSV
is a quick recovery operation, we should think about a more suitable mode for these patients. Methods: A total of 141 patients received TSV between January 2015 and July 2019: 81 patients received day surgery under caudal block (group A), and 60 received inpatient surgery under spinal anesthesia (group B). Operative time, postoperative hospital stay, hemospermia remission rate, magnetic resonance imaging (MRI) remission rate are compared. Visual analog scale (VAS) scores of groups were taken and compared at 2 time points: when there was pain during surgery (T1) and at the end of surgery (T2). Surgical methods of two groups are the same.

Results: The mean operative time of two groups are $34 \mathrm{~min}$ (group A) and $32 \mathrm{~min}$ (group B), and there was no statistical difference. Postoperative hemospermia remission rates are both $100 \%$ at 3 months, which at 6 months are $60 \%$ and $48 \%$, and there was no statistical difference. MRI remission at 3 months are $72 \%$ and $57 \%$, which has no statistical difference. Postoperative complications were mild in two groups like hematuria and dysuria which can relieve within one day, and there were no severe complications. Intraoperative pain was present in $18.5 \%(15 / 81)$ of group A. Their highest VAS score was 3 points, indicating mild pain, which did not influence the surgical process or postoperative recovery. The postoperative VAS scores were similar between the 2 groups. Group A did not require postoperative hospitalization, whereas the average postoperative hospitalization in group B was 2 days.

Conclusions: Seminal vesiculoscopy can be performed as a day surgery under caudal block, which has obvious advantages in accelerating postoperative recovery and shortening the hospital stay.

Keywords: Caudal block; day surgery; transurethral seminal vesiculoscopy (TSV); hemospermia; recurrence

Submitted Feb 28, 2020. Accepted for publication Sep 06, 2020.

doi: $10.21037 /$ tau-20-870

View this article at: http://dx.doi.org/10.21037/tau-20-870

\footnotetext{
^ ORCID: 0000-0003-3946-1501.
} 


\section{Introduction}

Hemospermia is a relatively common male reproductive system disease and can be induced by inflammation, ductal obstruction, stones and tumors (1). Intractable hemospermia is usually defined as a history longer than 3 months and persisting after more than 4 weeks antibiotics (2), it usually causes severe anxiety and influences the psychological and sexual quality of life (3). In recent years, transurethral seminal vesiculoscopy (TSV) has become a safe and effective method for diagnosis and management of intractable hemospermia, seminal vesicle calculi and ejaculatory duct obstruction, and itis widely confirmed by doctors in Asia, Europe and USA (4,5). Surgeons usually perform TSV under spinal anesthesia, and because of the effect of anesthesia, the postoperative hospital stay is 2-3 days $(6,7)$.

Recent years in China, enhanced recovery after surgery (ERAS) is widely concerned by doctors and hospital managers. Huge patient population make us to do great efforts to accelerate recovery after surgeries. The advantages of day surgery are that it can shorten the perioperative hospital stay and avoid hospitalization expenses. TSV is qualified for day surgery because of a natural cavity, a small surgical operation range, a short operative time, and a low incidence and severity of complications; in addition, patients can usually resume normal life 1 day after surgery (5). One major point of day surgery is the anesthesia mode, for example, surgeons usually choose local anesthesia for herniorrhaphy and caudal block for hemorrhoidectomy. As far as we know, there is no report about caudal block on TSV. Because caudal block can recover much faster than normal spinal anesthesia and it may cover the surgical region of TSV, we tried to apply it on day surgery.

The purpose of this retrospective study was to evaluate the efficacy and feasibility of TSV day surgery under caudal block compared to those of the conventional inpatient surgery.

We present the following article in accordance with the STROBE reporting checklist (available at http://dx.doi. org/10.21037/tau-20-870).

\section{Methods}

\section{Patients}

A total of 141 patients with intractable hemospermia received TSV treatment in the Department of Urology of our hospital between January 2015 and July 2019. Due to the establishment of day surgery ward recently in our hospital, 81 recent patients received day surgery under caudal block according to the choice of patients and doctors (group A). Sixty patients from previous years who received inpatient surgery under spinal anesthesia were assigned to inpatient surgery group (group B). The indications of TSV were the presence of more than 3 instances of hemospermia persistently or intermittently, more than 3 months of medical history, and at least 4 weeks of ineffective drug treatment (including antibiotics, nonsteroidal anti-inflammatory drugs, and single or combined application of Chinese patent drugs). All patients underwent preoperative prostate magnetic resonance imaging (MRI), blood prostate specific antigen (PSA) tests, and urine bacterial culture. Patients with abnormal PSA and suspected prostate cancer by MRI needed to further rule out prostate cancer without continuing this treatment. All operations were performed by the same senior surgeon and anesthesiologist. The preoperative baseline data are shown in Table 1. All procedures performed in this study were in accordance with the Declaration of Helsinki (as revised in 2013) and approved by the Ethics Committee of Xuanwu Hospital, Capital Medical University, China (registration ID: 2019033). Because of the retrospective nature of the research, the requirement for informed consent was waived.

\section{Day surgery method}

A mixture of $10 \mathrm{~mL} 1 \%$ lidocaine and $10 \mathrm{~mL} 0.25 \%$ ropivacaine was slowly injected (within 3-10 min) after caudal puncture. When pain was present during the surgery, intravenous analgesic drugs were administered, which were recorded along with the visual analog scale (VAS) pain score.

A 4.5-/6.5-Fr pediatric ureteroscope (Germany) was used in our hospital. The scope was first inserted to confirm the verumontanum utricle and enter under direct vision. If the ejaculatory duct opening was observed in the utricle, the endoscope was inserted further. If the opening was not observed, the scope tip was used to puncture the weakest part of the mucosa in the direction of the posterolateral wall of the utricle to enter the ejaculatory duct. The ejaculatory tube opening of some patients was located on both sides of the verumontanum utricle opening in the urethra. If the opening could not be found, the previous method was used. Semen in the vesicle was flushed until clear and bright. If there were combined stones, they could be flushed together. Larger stones were extracted through the urethra using a stone extraction basket or flushed after being powdered by a holmium laser. We usually explored the seminal vesicle of 
Table 1 Preoperative baseline data of patients in the 2 groups

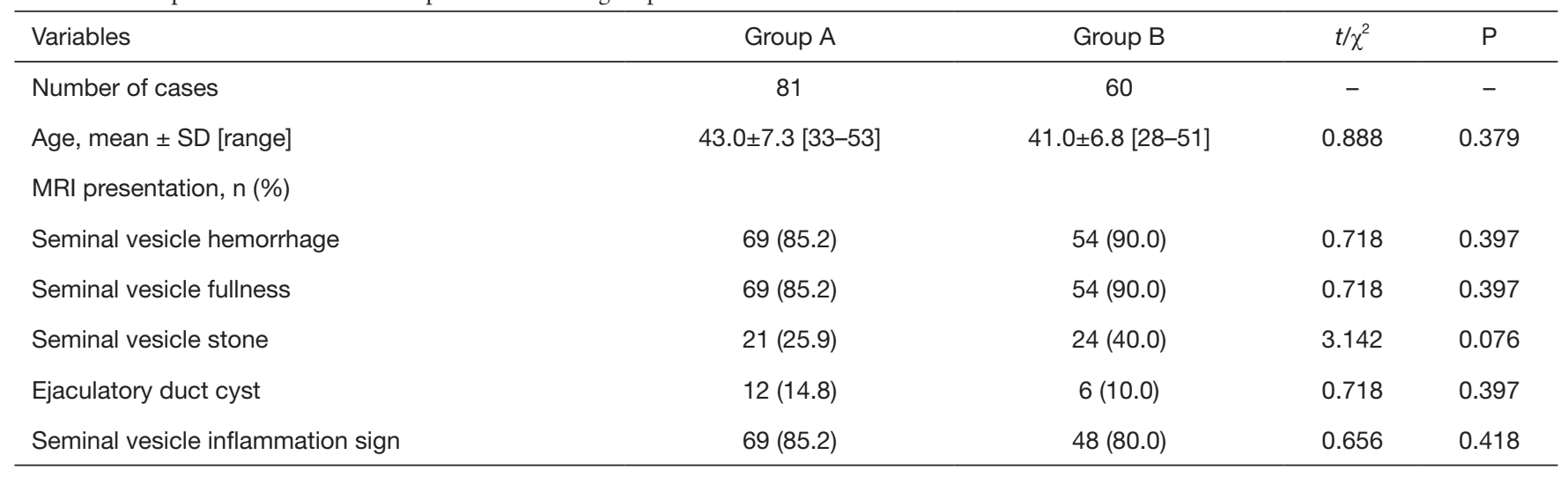

Group A: day surgery under caudal block; Group B: hospitalization surgery under intraspinal anesthesia. SD, standard deviation; MRI, magnetic resonance imaging.

the affected side based on postoperative MRI observations. The contralateral side usually did not receive conventional exploration, to avoid unnecessary injury and unnecessary operative time. No patients required indwelling urinary catheters after surgery. Patients were conventionally observed for several hours after surgery in a day ward and were discharged when the condition was stable.

\section{Inpatient surgery method}

Patients first received spinal anesthesia. Next, the same surgical method as in group A were adopted. The difference was that patients required postoperative placement of indwelling urinary catheters and returned to the inpatient ward. The catheters were removed the next day, and patients were discharged after 1-2 days.

\section{Follow-up and data collection}

Patients in both groups received oral antibiotics within 1 week after surgery to prevent infection. After 1 week, patients attempted to ejaculate by masturbation once every day for 3-5 days. Follow-up occurred once every month until at least 6 months after surgery. Prostate MRI was performed again 3 months postoperative. The major observation indicators included operative time, postoperative hospital stay, and VAS score. VAS scores were taken at 2 time points: when there was pain during surgery (T1) and at the end of surgery (T2). The minor observation indicators included the hemospermia remission rate 3 and 6 months postoperative, the MRI remission rate 3 months postoperative, and postoperative complications.

\section{Statistical methods}

Data were statistically analyzed using SPSS 22.0 software. Age, operative time, and hospital stay were compared between the 2 groups using the $t$-test. The incidence of hemospermia and the remission rate were compared using the $\chi^{2}$ test. VAS scores at different time points, which did not conform to normality and homogeneity of variances, were examined using the two-independent-sample MannWhitney $\mathrm{U}$-test. $\mathrm{P}<0.05$ indicated statistical significance.

\section{Results}

The baseline data between 2 groups were not significantly different (Table 1). The preoperative hemospermia incidence was $100 \%$. Based on MRI observation, the majority of intractable hemospermia patients had seminal vesicle hemorrhage on MRI (85.2\% in group A and 90\% in group B) combined with the presentation of seminal vesicle fullness and seminal vesicle inflammation. During surgery, only 6 cases prevented further seminal vesicle exploration because the endoscope could not enter the verumontanum utricle. The successful endoscope insertion rate was 95.7\%. Among MRI results of all patients, seminal vesicle hemorrhage was the most severe. The patient with the longest medical history was in group A, his age was 52 years, and his medical history of intractable hemospermia was 8 years (Figure $1 A, B, C, D$ ). The patient completed the surgical process smoothly under caudal block anesthesia. There was no pain during the surgery (T1) or at the end of the surgery (T2); therefore, the VAS scores were both 0 . The operative time was $40 \mathrm{~min}$, no postoperative indwelling 

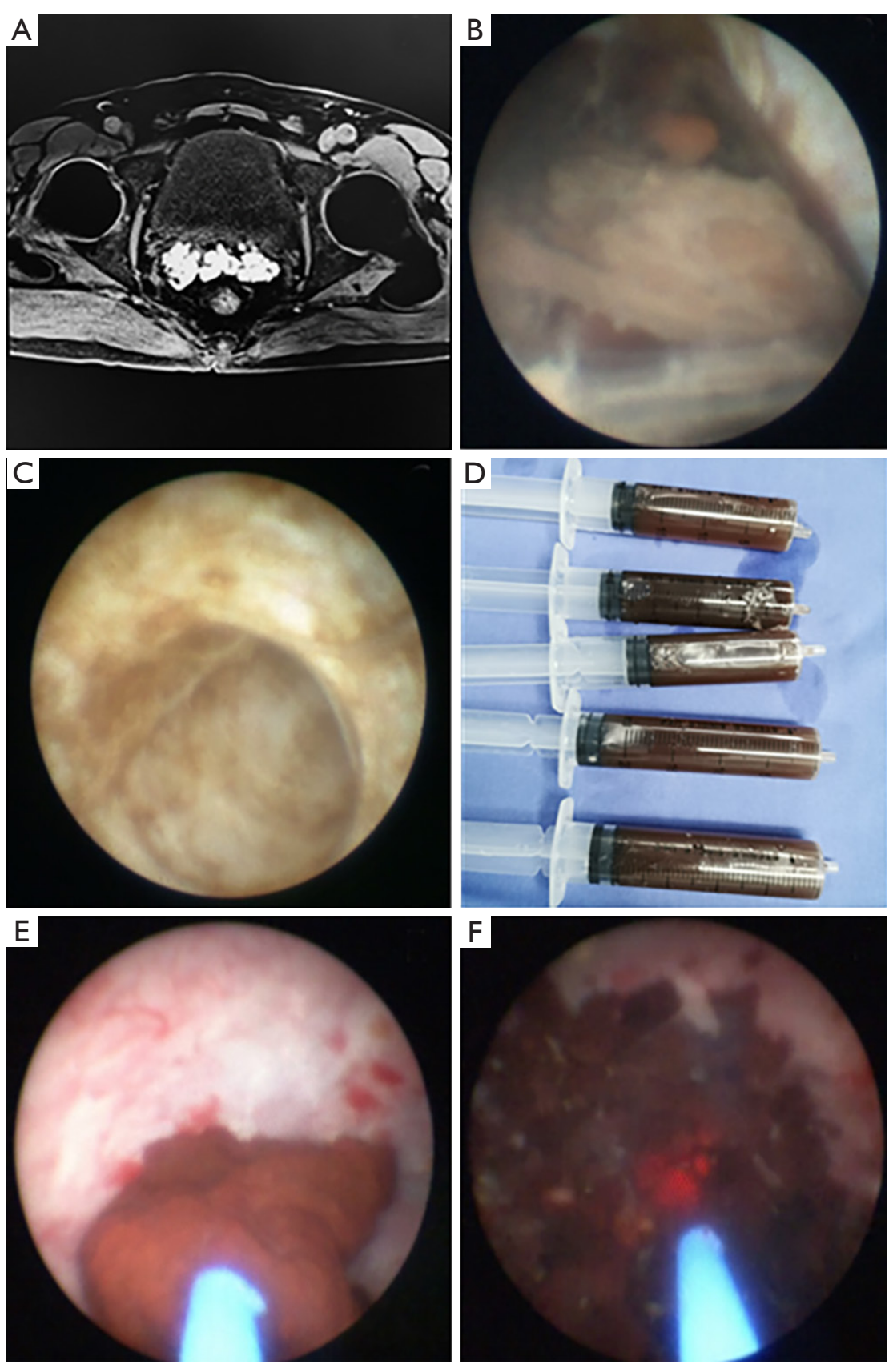

Figure 1 Patients with a large amount of blood clots (A,B,C,D) or stones (E,F) could complete the operations successfully within the validity period of caudal block in group A. (A) Bilateral seminal vesicles filled with blood clots on MRI; (B) endoscopic observation of a large amount of brown blood clots; (C) seminal vesicle after flushing; (D) postoperative specimens; (E) bilateral seminal vesicle stones; (F) result after lowenergy holmium laser lithotripsy at $0.5 \mathrm{~W} \times 30 \mathrm{~Hz}$. MRI, magnetic resonance imaging.

urinary catheter was required, and the patient was discharged on the same day. The patient with the longest operative time was also in group A. This patient had bilateral seminal vesicle stones and received holmium laser lithotripsy
(Figure $1 E, F)$; the operative time was $45 \mathrm{~min}$, including $28 \mathrm{~min}$ of laser lithotripsy. The patient complained of pain during lithotripsy; the VAS score was 2 points (T1), which was mild. Pain resolved after administration of intravenous 
Table 2 Comparison of operative time, VAS score, hospital stay, hemospermia remission rate, and MRI remission rate between the 2 groups

\begin{tabular}{|c|c|c|c|c|}
\hline Variables & Group A & Group B & $t / \chi^{2}$ & $\mathrm{P}$ \\
\hline Hospital stay time (days), mean \pm SD [range] & 0 & $1.9 \pm 0.8[1-3]$ & -10.180 & 0.000 \\
\hline \multicolumn{5}{|l|}{ VAS score, median [min, max] } \\
\hline T1 & $0[0,3]$ & $0[0,0]$ & - & 0.044 \\
\hline $\begin{array}{l}\text { Hemospermia remission at postoperative } 3 \\
\text { months, } \mathrm{n}(\%)\end{array}$ & $81(100.0)$ & $60(100.0)$ & - & - \\
\hline $\begin{array}{l}\text { Hemospermia remission at postoperative } 6 \\
\text { months, } \mathrm{n}(\%)\end{array}$ & $60(74.1)$ & $48(80.0)$ & 0.675 & 0.411 \\
\hline MRI remission at postoperative 3 months, $\mathrm{n}(\%)$ & $72(100.0)$ & $57(100.0)$ & - & - \\
\hline
\end{tabular}

VAS scores that did not conform to normality and homogeneity variances were examined using the two-independent-sample MannWhitney U-test. T1: intraoperative VAS pain score; T2: VAS pain score at the end of surgery. Group A: day surgery under caudal block; group B: hospitalization surgery under intraspinal anesthesia. VAS, visual analog scale; MRI, magnetic resonance imaging; SD, standard deviation.

analgesic drugs. The surgery was completed smoothly, and the patient was discharged on the same day after surgery. A total of 15 patients in group A had pain, and no one in group B had pain. The patient with the highest VAS score during surgery (T1) was in group A. This patient had pain when the endoscope entered the seminal vesicle; the VAS score was 3 points, which was mild. The surgery was completed smoothly after intravenous analgesic drugs were administered by the anesthesiologist, and the patient was discharged on the same day. Comparison of VAS scores between the 2 groups showed that the average T1 score of group A was higher than that of group $\mathrm{B}(\mathrm{P}<0.05)$, whereas the VAS scores of patients in these 2 groups at $\mathrm{T} 2$ were both 0 . The average operative time in both groups was 34 and 32 min $(\mathrm{P}>0.05)$. Regarding hospital stay time, group A did not require hospitalization because of caudal block, whereas the average hospital stay of group B was 2 days. Comparisons of operative time, VAS score, and postoperative hospital stay between the 2 groups are shown in Table 2.

Regarding follow-up, 141 patients were all followed up for at least 6 months after surgery. One hundred and twentynine patients underwent MRI at postoperative 3 months. The hemospermia remission rate in group A was lower than that in group B, but the difference did not reach statistical significance. The seminal vesicle hemorrhage remission rates on MRI was $100 \%$ in each group (Table 2). The results during follow-up showed that, after the initial 3-5 intensive ejaculations within a week postoperative, hemospermia of all patients was relieved without recurrence for 3 months.
In other words, hemospermia had a $100 \%$ remission in all patients 3 months postoperative. Twenty-one patients in group A and 12 patients in group B complained of the recurrent between 3 and 6 months postoperative. These patients occurred again after 3 months or longer, but the degree was less than before. Four patients did not undergo another MRI examination for personal reasons (9 in group A and 3 in group B). The other 129 patients did not have seminal vesicle hemorrhage on MRI examinations. These 2 groups did not have severe complications, including epididymitis, retrograde ejaculation, rectal injury, urinary incontinence, and erectile dysfunction.

\section{Discussion}

To our knowledge, there is no relevant report on TSV day surgery. We made this initial attempt and retrospectively summarized the feasibility of its application. Our experience reported here can lay the foundation for prospective studies with large samples sizes in the future. Chen et al. (8) reported a retrospective study with the largest number of intractable hemospermia patients treated with TSV so far and confirmed the effectiveness and safety of TSV. The average operative time was $21 \mathrm{~min}$, the surgical success rate was $90.9 \%$, and the hemospermia remission rate was $85.0 \%$; which were close to results of our study. The relevant studies in recent years have made it obvious that, with the growing experience and technical skill of surgeons and the application of novel seminal vesiculoscopes and lithotripsy 
apparatuses, TSV operative time has become shorter (the average reported time is $30 \mathrm{~min})(6,9)$. In addition, the postoperative recovery time has become faster. Even for patients who receive laser lithotripsy, they can completely recover within 1 day after surgery (5). The operative range of seminal vesiculoscopy is very limited, and the likelihood of severe postoperative complications is also very low.

Day surgery mode can effectively integrate medical resources, increase resource utilization efficiency, relieve conflicts between the supply and demand of medical resources, shorten the waiting time of patients before admission, shorten hospital stays, increase the bed utilization rate, and reduce medical expenses (10). Day surgery in many large, third-grade, class-A hospitals in China already accounts for more than $20 \%$ of elective surgeries. With the growing understanding of seminal vesiculoscopy technology in recent years, it has come to be characterized by a short operative time, small operative range, little trauma, and fast recovery, thus meeting the requirements for day surgery (11).

Postoperative anesthesia recovery is an important step in day surgery. Current relevant reports on seminal vesiculoscopy all used general anesthesia or spinal anesthesia. However, unexpected penile erection during general anesthesia greatly influences the operation, also the anesthesiologists always show few methods to solve it. The application of 4.5/6.5-Fr pediatric ureteroscopes in recent years has greatly increased the success rate of the insertion of seminal vesiculoscopes. However, the body of the endoscope is slim, and the hardness of the body of the endoscope is lower than that of a common ureteroscope; therefore, penile erection can bend and damage the instrument. Although some methods can address intraoperative erections (12), not all have been satisfactory in our practice, and the surgery have to stop sometimes. Therefore, general anesthesia is not used during seminal vesiculoscopy in our center. Although spinal anesthesia avoids the intraoperative erections, patients always have to stay in the hospital for 1-2 days because of anesthesia effects and postoperative catheter indwelling. Caudal block can cover sacrococcygeal nerves that innervate the whole perineal area, including the perianal area, rectum, and prostate. It is mainly used in perianal surgery and pediatric perineal surgery and can be used in urological techniques, including cystoscopy and transrectal prostate biopsy. Its application is even more extensive in Japanese reports (13). Wang et al. recommended that the dose of anesthetic drugs in caudal block during transrectal prostate biopsy not be lower than $15 \mathrm{~mL}$, and the recommended dose was $20 \mathrm{~mL}$, such as $20 \mathrm{~mL} 0.5 \%$ lidocaine plus $75 \mu \mathrm{g}$ fentanyl (14). In this study, we used the mixture of $10 \mathrm{~mL} \mathrm{1 \%} \mathrm{lidocaine} \mathrm{plus} 10 \mathrm{~mL} 0.25 \%$ ropivacaine and achieved satisfactory anesthetic effects.

There is still no relevant literature on TSV under caudal block. The results of our first attempt showed that operative time, postoperative hemospermia remission rate, and surgical complications were not different from those in the spinal anesthesia group. Postoperative VAS scores (T2) for both groups were all 0, so there was no significant difference. A total of 15 cases in group A had intraoperative pain, for an incidence of $18.5 \%(15 / 81)$, which was close to that in the report of Ikuerowo et al. (15). Analysis of possible causes of this type of pain showed that, in addition to sacrococcygeal nerve groups, the prostate is also innervated by some preaortic nerve groups (16). Although the intraoperative VAS score (T1) for group A was higher than that for group $\mathrm{B}(\mathrm{P}=0.044)$, the average score was close to 0 , the highest VAS was only 3 points, and pain was all mild; thus, the clinical significance was low. Pain was completely relieved after providing 1 injection of an intravenous analgesic drug, such as oxycodone hydrochloride. Therefore, it did not influence subsequent surgery and prognosis. The advantages of caudal block are fast recovery, little trauma, simple operation, which reduce the effect of anesthesia on the postoperative recovery rate. No patients in this study had anesthesia complications after surgery. Compared to spinal anesthesia, the biggest advantage of caudal block is unrestricted lower limb activity, which makes patients able to walk home. Combining caudal block with the day surgery mode, patients could be discharged right after surgery.

The limitation of this study was retrospective. Notably, baseline characteristics and preoperative imaging presentations did not differ between the 2 groups. In the future, we will perform prospective study for further exploration and validation.

TSV performed as a day surgery under caudal block was feasible. The surgical efficacy was not different from that of inpatient surgery. The advantages were faster recovery and no hospital stay. Therefore, this surgery mode can be implemented in large hospitals that have the capacity for day surgery.

\section{Acknowledgments}

We thank Prof. Hui Jiang, Prof. Kai Hong from Department of Andrology, Peking University Third Hospital, and Prof. Hong-Jun Li from Department of Urology, Peking Union Medical College Hospital for their valuable proposals to our work.

Funding: None. 


\section{Footnote}

Reporting Checklist: The authors have completed the STROBE reporting checklist. Available at http://dx.doi. org/10.21037/tau-20-870

Data Sharing Statement: Available at http://dx.doi. org/10.21037/tau-20-870

Conflicts of Interest: All authors have completed the ICMJE uniform disclosure form (available at http://dx.doi. org/10.21037/tau-20-870). The authors have no conflicts of interest to declare.

Ethical Statement: The authors are accountable for all aspects of the work in ensuring that questions related to the accuracy or integrity of any part of the work are appropriately investigated and resolved. All procedures performed in this study were in accordance with the Declaration of Helsinki(as revised in 2013) and approved by the Ethics Committee of Xuanwu Hospital, Capital Medical University, China (registration ID: 2019033). Because of the retrospective nature of the research, the requirement for informed consent was waived.

Open Access Statement: This is an Open Access article distributed in accordance with the Creative Commons Attribution-NonCommercial-NoDerivs 4.0 International License (CC BY-NC-ND 4.0), which permits the noncommercial replication and distribution of the article with the strict proviso that no changes or edits are made and the original work is properly cited (including links to both the formal publication through the relevant DOI and the license). See: https://creativecommons.org/licenses/by-nc-nd/4.0/.

\section{References}

1. Ahmad I, Krishna NS. Hemospermia. J Urol 2007;177:1613-8.

2. Hu JC, Chen CS. Transurethral seminal vesiculoscopy acts as a therapeutic investigation for intractable hemospermia: Step-by-step illustrations and single-surgeon experience. Int J Urol 2018;25:589-95.

3. Raviv G, Laufer M, Miki H. Hematospermia--the added value of transrectal ultrasound to clinical evaluation: is transrectal ultrasound necessary for evaluation of hematospermia? Clin Imaging 2013;37:913-6.

4. Christodoulidou M, Parnham A, Nigam R. Diagnosis and management of symptomatic seminal vesicle calculi. Scand
J Urol 2017;51:237-44.

5. Zaidi S, Gandhi J, Seyam O, et al. Etiology, Diagnosis, and Management of Seminal Vesicle Stones. Curr Urol 2019;12:113-20.

6. Zhang W, Xiao G, Qin S, et al. An Innovative Technique of Transurethral Seminal Vesiculoscopy with Ultrasonic Lithotripter for Severe, Persistent Hematospermia. J Endourol 2017;31:1277-82.

7. Xue RZ, Tang ZY, Chen Z, et al. Clinical outcomes of transperitoneal laparoscopic unroofing and fenestration under seminal vesiculoscopy for seminal vesicle cysts. Asian J Androl 2018;20:621-5.

8. Chen R, Wang L, Sheng X, et al. Transurethral seminal vesiculoscopy for recurrent hemospermia: experience from 419 cases. Asian J Androl 2018;20:438-41.

9. Oh TH, Seo IY. Endoscopic Treatment for Persistent Hematospermia: A Novel Technique Using a Holmium Laser. Scand J Surg 2016;105:174-7.

10. Mitchell $M$. Home recovery following day surgery: a patient perspective. J Clin Nurs 2015;24:415-27.

11. Anderson T, Walls M, Canelo R. Day case surgery guidelines. Surgery (Oxford) 2017;35:85-91.

12. Guler G, Sofikerim M, Ugur F, et al. Intravenous dexmedetomidine for treatment of intraoperative penile erection. Int Urol Nephrol 2012;44:353-7.

13. Wang N, Fu Y, Ma H, et al. Advantages of caudal block over intrarectal local anesthesia plus periprostatic nerve block for transrectal ultrasound guided prostate biopsy. Pak J Med Sci 2016;32:978-82.

14. Wang J, Zhou H, An W, et al. Effective Concentration of Lidocaine Plus Fentanyl for Caudal Block in Patients Undergoing Transrectal Ultrasound Guided Prostate Biopsy. Pain Res Treat 2016;2016:5862931.

15. Ikuerowo SO, Popoola AA, Olapade-Olaopa EO, et al. Caudal block anesthesia for transrectal prostate biopsy. Int Urol Nephrol 2010;42:19-22.

16. Horinaga $M$, Nakashima J, Nakanoma T. Efficacy compared between caudal block and periprostatic local anesthesia for transrectal ultrasound-guided prostate needle biopsy. Urology 2006;68:348-51.

Cite this article as: Cui B, Wu JT, Xu JJ, Ou TW. Efficacy and feasibility of day surgery using transurethral seminal vesiculoscopy under caudal block anesthesia for intractable hemospermia. Transl Androl Urol 2020;9(6):2493-2499. doi: $10.21037 /$ tau-20-870 